\title{
Une jeunesse (in)visible : les « Neets vulnérables » de la Garantie jeunes
}

Young vulnerable in the shadow : the French Garantie jeunes

Julie Couronné et François Sarfati

\section{(2) OpenEdition}

\section{Journals}

Édition électronique

URL : http://journals.openedition.org/travailemploi/7905

DOI : 10.4000/travailemploi.7905

ISSN : 1775-416X

Éditeur

DARES - Ministère du Travail

Édition imprimée

Date de publication : 1 janvier 2018

Pagination : 41-66

ISSN : 0224-4365

Référence électronique

Julie Couronné et François Sarfati, « Une jeunesse (in)visible : les « Neets vulnérables » de la Garantie jeunes », Travail et Emploi [En ligne], 153 I janvier-mars 2018, mis en ligne le 11 juillet 2019, consulté le 05 septembre 2019. URL : http://journals.openedition.org/travailemploi/7905; DOI : 10.4000/ travailemploi.7905 


\title{
Une jeunesse (in)visible : les « Neets vulnérables » de la Garantie jeunes
}

\author{
Julie Couronné, François Sarfati ${ }^{* *}$
}

\begin{abstract}
À partir d'une enquête ethnographique menée dans trois missions locales mettant en œuvre la Garantie jeunes, cet article analyse la catégorie de « Neets vulnérables », qui constitue la cible du dispositif. Cette catégorie est traversée par deux processus contradictoires. Le premier participe à rendre visible une jeunesse issue des classes populaires les plus démunies, qui correspond bien à la cible : ces jeunes cumulent un ensemble de difficultés depuis le plus jeune âge et leurs ressources économiques sont très réduites. Le second processus à l'œuvre invisibilise en revanche une partie des difficultés rencontrées par ces jeunes. La définition de la cible par la négative induite par l'expression « Neets vulnérables » ne prend pas en considération leurs expériences de travail et ne dit rien de leur rapport à l'emploi, les réduisant ainsi à des jeunes « sans expérience », « sans travail », « sans diplôme ». Or, la réalité de leur situation au moment où ils entrent à la Garantie jeunes est bien plus complexe, comme le montrent les quarante parcours reconstitués.
\end{abstract}

Droposée aux jeunes âgés de 16 à 25 ans qui ne sont ni en études, ni en emploi, ni Pen formation - les « Neets », (Not in education, employment, or training) -, la Garantie jeunes cible les plus vulnérables d'entre eux. Son objectif est «d'amener les jeunes en situation de grande précarité vers l'autonomie par l'organisation d'un parcours d'accompagnement global, social et professionnel, vers et dans l'emploi ou la formation $^{1} \gg$. Ce dispositif comporte deux éléments pensés comme complémentaires. Le premier réside dans un accompagnement individuel et collectif des jeunes organisé dans et par les missions locales. La phase inaugurale consiste à accueillir les destinataires du dispositif par groupes de quinze pendant six semaines. Des ateliers leur sont proposés tout au long de la journée (simulation d'entretien d'embauche, rédaction de curriculum vitæ, etc.). Par ailleurs, les jeunes sont accompagnés de manière

\footnotetext{
* Centre d'études de l'emploi et du travail, Cnam (Lise-CNRS), julie.couronne@cnam.net.

** Centre d'études de l'emploi et du travail, Cnam (Lise-CNRS), francois.sarfati@lecnam.net.

1. Article du décret $n^{\circ} 2013-880$ du $1^{\text {er }}$ octobre 2013 relatif à l'expérimentation de la « Garantie jeunes ».
} 
individuelle, par les conseillers d'insertion de la Garantie jeunes. Le second élément est une allocation d'un montant mensuel de 471 euros (en 2017). Sensiblement plus importante que celle proposée par d'autres dispositifs comme le Civis ${ }^{2}$ (250 euros), elle peut être cumulée avec les revenus du travail.

Ainsi, dès 2013, la Garantie jeunes participe à l'émergence sur la scène publique de la catégorie de « Neets vulnérables ». Bon nombre de textes produits par la Commission européenne mobilisent l'expression « Neets ». Cette littérature appréhende les vulnérabilités juvéniles à partir du niveau de chômage des jeunes. De plus, cet indicateur a fait l'objet de critiques fortes, notamment parce qu'il écarte de nombreux jeunes ni en emploi, ni en formation, ni en recherche d'emploi (FurLONG, 2007). En 1994, une étude est consacrée à des adolescents de 16 et 17 ans qui ne sont pas en formation et sont trop jeunes pour percevoir une allocation-chômage (ISTANCE et al., 1994). Cette étude, dont le titre donnera le nom à la catégorie Neets, renvoie à l'existence de jeunes sans statut, les Status ZerO. Six ans plus tard, en 2010, la Commission européenne introduit un indicateur statistique relatif aux «sans-statut», permettant d'identifier des jeunes âgés de 15 à 29 ans considérés comme Neets. La démarche consiste à rendre visibles des situations de jeunesse qui jusque-là restaient dans l'ombre. Outre les jeunes au chômage, la littérature européenne évoque des jeunes découragés, des inactifs et plus largement des jeunes désœuvrés. En Grande-Bretagne, le programme du New Labour consistant à proposer une politique de «Welfare to Work» mobilise l'expression. Les jeunes Neets sont alors considérés comme responsables de leur situation. Le programme du «New Deal» cherche à mettre au travail des jeunes exclus (EuRofound, 2012). Puis, en juin 2012, le Conseil européen encourage les États membres à « intensifier les efforts visant à augmenter l'emploi des jeunes, et notamment à améliorer la première expérience professionnelle des jeunes et leur participation au marché du travail, l'objectif étant que, en l'espace de quelques mois suivant leur sortie de l'école, ils se voient proposer un emploi de bonne qualité, une formation continue, un apprentissage ou un stage, pouvant bénéficier du soutien du Fonds social européen ; à élaborer et mettre en œuvre des politiques efficaces visant à lutter contre la pauvreté ainsi qu'à soutenir les groupes vulnérables » (CONSEIL EUROPÉEN, 2012, p. 8). Le Conseil de l'Union européenne adopte ensuite une recommandation visant à établir une « garantie pour la jeunesse », qui cible 7,5 millions de Neets, avec l'objectif de leur « proposer un emploi de qualité, une formation continue, un apprentissage ou un stage dans les quatre mois suivant la perte de leur emploi ou leur sortie de l'enseignement formel » (CONSEIL DE L’UNION EUROPÉENNE, 2013).

Dans le cas français, la Garantie jeunes s'intègre dans un effort coordonné de proposer des solutions aux jeunes en difficulté. La France se saisit de l'opportunité offerte par l'Europe de financer un dispositif pour l'emploi des jeunes. Un rapport propose un cadrage permettant d'opérationnaliser la catégorie Neets en délimitant des

2. Contrat d'insertion dans la vie sociale, le Civis est un dispositif également mis en place dans les missions locales, destiné aux jeunes âgés de 18 à 25 ans. Depuis la loi Travail d'août 2017, il est remplacé par le « parcours contractualisé d'accompagnement vers l'emploi et l'autonomie ». 
« critères d'accès » (WARGON, GURGAND, 2013 ${ }^{3}$ ). Cette dernière est ensuite reprise par les concepteurs de la Garantie jeunes, qui y ajoutent un critère de vulnérabilité économique. C'est à ce moment-là que la catégorie « Neets vulnérables » est officiellement créée. Politique active du marché du travail (GaUtié, 2015), la Garantie jeunes vise l'insertion professionnelle de jeunes en situation de vulnérabilité économique et sociale. La stratégie retenue est celle dite du work first. À rebours de l'idée selon laquelle il conviendrait de régler les « freins périphériques à l'emploi ${ }^{4}$ » avant d'aider les chômeurs à accéder au marché du travail, le work first consiste à mettre les personnes en situation de travail, quelle que soit l'activité, quel que soit le statut (stage, CDD, intérim, etc.), avant toute chose. Cette philosophie d'intervention suppose que l'accès au travail en premier lieu permettra, dans un second temps, de résoudre les autres difficultés rencontrées par les personnes.

Outre l'analyse des effets du dispositif sur ses destinataires, l'objectif de cet article est de contribuer à la réflexion sur les opérations de catégorisation. En effet, la production de catégories statistiques par l'État met en forme le monde social et reconnaît l'existence de groupes sociaux (DESROSIÈRES, 1993). Des acteurs sociaux se mobilisent et œuvrent à l'émergence de problèmes publics reposant sur ce mode de catégorisation (GUSFIELD, 2009). Il s'agit alors d'analyser la tension qui existe autour de la catégorie « Neets vulnérables » entre la manière dont les concepteurs se représentent ces jeunes et leurs caractéristiques sociales. Cette catégorie - comme toutes les catégories - a ses limites. Elle est traversée par deux processus contradictoires. Le premier contribue à rendre visible une jeunesse issue des classes populaires les plus démunies et à la doter d'un droit sans précédent. L'un des résultats importants de notre enquête de terrain (encadré 1) montre que les jeunes rencontrés correspondent bien à la cible attribuée au dispositif : il s'agit de jeunes cumulant un ensemble de difficultés depuis le plus jeune âge et dont les ressources économiques sont très réduites. En ce sens, la catégorie « Neets vulnérables » est opératoire car la cible est atteinte. En revanche, le second processus à l'œuvre invisibilise une partie des difficultés rencontrées par les jeunes. La définition de la cible par la négative ne prend pas en considération les expériences de travail de ces jeunes et ne dit rien sur leur rapport à l'emploi, les réduisant ainsi à des jeunes « sans expérience », « sans travail », « sans diplôme ». Or, la réalité de leur situation au moment où ils entrent à la Garantie jeunes est bien plus complexe et diverse, comme le montrent les quarante parcours de jeunes que nous avons reconstitués. Enfin, en mettant la focale sur deux « jeunes Garantie jeunes » aux situations contrastées, l'article rend compte de l'effet de l'accompagnement sur les parcours des individus. Si la catégorie « Neets vulnérables » ignore le passé professionnel des jeunes, les conseillers mobilisent leur expérience professionnelle antérieure pour les accompagner dans et vers l'emploi.

3. «Il s'agit des jeunes majeurs de 18-25 ans qui ne sont «ni en emploi, ni en éducation, ni en formation » (Neet) et dont les ressources ne dépassent pas le plafond du RSA (483€ pour une personne seule). Ces jeunes peuvent être décohabitants sans soutien familial ou cohabitants mais sans autre soutien familial. » (WARGON, GURGAND, 2013).

4. L'expression est régulièrement mobilisée dans le vocabulaire indigène pour regrouper les problèmes de formation, de logement ou encore de mobilité, qui empêchent d'accéder à l'emploi. 


\section{ENCADRÉ 1}

\section{Méthode et conditions d'enquête ${ }^{1}$}

L'enquête de terrain a été réalisée entre mars 2015 et mars 2016 au sein de trois missions locales accueillant des populations de jeunes contrastées, ces derniers évoluant dans des milieux urbains (mission locale orange) et semi-urbains (mission locale verte et mission locale violette). La méthode utilisée est ethnographique (WEBER, 2009) et mobilise plusieurs techniques : entretiens semi-directifs, observations participantes, entretiens collectifs, analyse de documents récoltés sur le terrain et photographies.

Dans un premier temps, 60 entretiens semi-directifs ont été menés auprès d'une partie des jeunes ayant intégré la Garantie jeunes, avec des entrées dans le dispositif situées entre $n+1$ jour et $n+134$ jours. Ces entretiens ont eu pour principal objectif de reconstituer les parcours biographiques des jeunes dans toutes leurs dimensions (scolaire, familiale, résidentielle, institutionnelle). Par ailleurs, une dizaine d'entretiens a été réalisée auprès des directeurs des missions locales étudiées et des acteurs institutionnels issus du milieu de l'insertion sociale et professionnelle. Parallèlement à la conduite de ces entretiens, 20 journées d'observation ont été effectuées au cours de l'accompagnement collectif inaugural. Ces dernières ont consisté à observer les groupes de jeunes et à participer aux ateliers proposés tels que « atelier théâtre », « lettre de motivation », «SST » (formation de sauveteur secouriste au travail), « atelier relooking », etc.

Dans un deuxième temps, l'enquête a pris la forme d'entretiens collectifs (trois au total) et d'observations de jeunes en entreprise, dans le cadre de stages (trois journées).

Enfin, 40 entretiens téléphoniques ont été réalisés auprès des jeunes rencontrés au cours de la première vague d'entretiens. Ils ont permis de retracer le parcours des jeunes au sein du dispositif et de faire le point sur leur situation familiale, résidentielle et professionnelle. Par ailleurs, 40 portraits biographiques ont ainsi été construits à partir de l'ensemble des données recueillies à différents moments (entretiens collectifs, entretiens auprès des jeunes mais également auprès des professionnels, observations) et retracent de manière dynamique le parcours des jeunes.

Ce protocole d'enquête repose sur la volonté de créer des conditions d'échange qui prennent en compte à la fois les caractéristiques sociales des enquêtés et leur vécu (Couronné, 2016). Dans certaines situations, l'entretien collectif a été un moyen très efficace pour dépasser les échanges aux airs de « question-réponse » qui caractérisaient les premiers entretiens réalisés dans des conditions « classiques »-face à face de longue durée avec à l'appui un guide d'entretien, un enregistrement du propos ainsi qu'un effort de verbalisation et d'introspection demandé au jeune. Les trois entretiens collectifs ont été animés par deux sociologues de façon moins formelle que les entretiens individuels. L'échange ne s'est pas appuyé sur un guide d'entretien, mais sur des photographies que les jeunes avaient prises quelques minutes avant l'entretien, avec la consigne d'illustrer leur expérience au sein du dispositif. Ce procédé ludique les a amusés et les a impliqués dans la démarche de l'entretien collectif. Les relances pour les faire parler ont été formulées

1. Cette enquête a été réalisée avec Marie Loison-Leruste, maîtresse de conférences à l'université Paris 13 et membre du Ceral. Les résultats exposés ici n'engagent que les auteur·trice·s. 
en s'appuyant sur le registre des « joutes oratoires » (LEPOUTRE, 1997), de la taquinerie bienveillante et de l'échange de vannes. La mise en place volontaire de ces conditions d'échange a permis d'ouvrir une « fenêtre de confiance » (BIZEuL, 2007, p. 84) et d'accéder à un discours beaucoup plus critique que celui recueilli en entretien individuel (CoURONNÉ, 2017).

\section{Une cible atteinte}

Les jeunes que nous avons rencontrés dans les missions locales ne sont, au moment de leur entrée dans le dispositif, «ni en emploi, ni en formation, ni en stage ». De ce fait, ils correspondent bien à la cible visée par la Garantie jeunes. En effet, leurs biographies sont marquées par des ruptures scolaires, des difficultés économiques et un parcours hyper-institutionnalisé.

\section{Des ruptures scolaires}

Leurs difficultés scolaires apparaissent majoritairement pendant les années de collège. En entretien, les jeunes insistent sur leurs problèmes relatifs à l'apprentissage, leur comportement « agité » en cours et leur tendance à s'absenter de l'école de manière régulière. Certains racontent également avoir été victimes de harcèlement pendant plusieurs années. Ne supportant plus les moqueries et les menaces quotidiennes, ils mettent fin à leur scolarité. C'est par exemple le cas de Michaela ${ }^{5}$ [E27] :

«En fait, j'étais déscolarisée pour des menaces de mort. Moi, j'ai pas un parcours toujours facile. J'ai été déscolarisée pour menaces de mort. Le lycée n'a rien fait pour m'aider, et on devait changer de classe, parce que c'était un camarade de ma classe. Il fallait que je lui fasse ses devoirs, que je lui fasse tout, en fait. Et j'ai refusé et il m'a menacée de mort, et il a été viré trois, quatre jours, c'est tout. Et après il est revenu. [...] Et moi, après les vacances, au moment des vacances de novembre, je finis septembre, octobre, en novembre j'étais hospitalisée quatre jours avant les vacances. Et après les vacances, le seuil du lycée, j'ai pas pu le refranchir. »

(Michaela [E27], 21 ans, sans diplôme)

La plupart des enquêtés se distinguent par leur faible niveau de qualification (niveau CAP et BEP) et par des arrêts de scolarité précoces, avant 16 ans, l'âge de la fin d'obligation scolaire. Nombreux sont ceux qui sont sortis du système scolaire sans diplôme. Seulement deux jeunes sur les soixante constituant la population enquêtée

5. Les numéros indiqués entre crochets correspondent aux numéros d'entretien. L'ensemble des entretiens menés auprès des jeunes et des professionnels ainsi que les caractéristiques sociales des enquêtés sont répertoriés dans un tableau récapitulatif placé en annexe. 
ont obtenu un baccalauréat général, et ils sont rares à être titulaires d'un baccalauréat professionnel ou technologique. Une partie d'entre eux décrivent des «erreurs d'aiguillage » (BECQUEMIN, MARDON, CouRONNÉ, 2012) pour qualifier leurs parcours scolaires. Ces erreurs « ne se réduisent pas aux problèmes d'orientation produits par le système scolaire bien qu'ils soient massivement évoqués. Le terme renvoie plus largement à l'impression exprimée par les jeunes de ne pas être à leur place ou à celle qu'ils souhaiteraient, pour des raisons variées » (ibid.). Les enquêtés rendent compte d'orientations scolaires subies ou déçues, souvent incomprises.

«Ben déjà mon collège, je l'ai pas terminé jusqu'en troisième, parce que à partir de la quatrième, je suis partie au lycée professionnel, pour faire une troisième DP6. Et ce qui a fait que j'ai pris le bac pro commerce, c'est que j'ai pas eu le choix : j'ai pas trouvé d'apprentissage, donc on m'a mis directement sur le bac pro commerce [...] Si t'as pas d'entreprise, tu peux pas rentrer en apprentissage. »

(Laura [E5], 20 ans, niveau CAP)

Dans la plupart des cas, les jeunes ne connaissent pas la raison de leur orientation vers des dispositifs spécialisés, tels que la Segpa :

«En fait au début, moi en sixième, j'étais au collège en général. Et après mon proviseur du collège des ****, en fait il m'a... il m'a viré, parce que j'étais trop nul en général, et qu'il fallait me mettre en Segpa ».

(Neil [E16], 19 ans, sans diplôme)

Ils sont nombreux à déclarer avoir « arrêté » ou « abandonné », ou à avoir été exclus du système scolaire sur décision d'un conseil de discipline comme Amidou [E20] :

«Maternelle, j'ai fait ma... petite section, moyenne section, grande section, j'ai tout fait. Et je suis arrivé au CP. CP j'ai redoublé, parce que je connaissais rien. Je connaissais que des gros mots, rien du tout, donc j'ai redoublé. J'ai fait toute ma primaire jusqu'en CM2. Et je suis allé au collège, là, j'ai fait toute la scolarité mais j'ai pas eu mon brevet, en troisième. Après je suis allé à ****,j'ai fait un CAP installateur sanitaire. Et j'ai redoublé, parce que j'étais souvent absent, ils pensaient que ça m'intéressait pas... je faisais n'importe quoi, un peu. J'ai redoublé et j'ai fait une autre année, et ils m'ont donné que des avertissements, nanana, et après ils m'ont viré. »

(Amidou [E20], 20 ans, sans diplôme)

Certains jeunes expriment des regrets à l'égard de leur trajectoire scolaire et de leur comportement à l'école. Enfin, comme Laura [E5], citée plus haut, plusieurs jeunes dénoncent la difficulté d'obtenir un contrat d'apprentissage auprès d'un employeur lorsqu'ils intègrent une formation en alternance. Une fois que la scolarité des jeunes rencontrés s'interrompt, une partie d'entre eux se redirige vers d'autres dispositifs comme ceux des Écoles de la deuxième chance ou d'autres organismes de formation. 


\section{Des difficultés économiques héritées}

À ces difficultés liées à l'école ou à un parcours antérieur de formation s'ajoutent des difficultés économiques largement héritées. L'usage de l'allocation en est un bon révélateur. Ainsi, de nombreux jeunes l'utilisent pour combler des dettes accumulées par des impayés de loyer, des soins ou des emprunts.

« Là je suis tout seul à la maison, y'a plus rien dans le frigo, il serait peut-être temps de faire les courses ! Et euh... en fait je suis obligé de laisser mon compte dans le positif pendant 48 heures avant de pouvoir utiliser ma carte. Du coup fatalement ça fait... [Mon père] dans sa grande bonté il s'est dit : « De toute façon tu auras de l'argent le $15 \ldots$.. Je fais : « Oui enfin, le 15 ! » Il est parti le 2, enfin non il est parti le 3, il me dit: « Oui t'inquiète t'auras de l'argent le 15 ! » J'ai dit : «Le 15 ! Le frigo il est vide hein ! Jusqu'au 15 le frigo il est vide ! » Mon père il est comme ça, j'ai l'habitude maintenant. »

(Jacques [E55], 23 ans, sans diplôme)

« J'ai été... j'ai eu des problèmes, parce que j'habite seule, moi, j'ai un appartement, j'ai eu des problèmes avec mon bailleur, j'ai eu des impayés, donc j'ai été voir l'assistante sociale. J'avais pas droit au RSA, vu mon âge [...]. Donc en fouillant sur Internet, parce que j'étais désespérée, je cherchais au moins une aide, parce qu'il fallait avoir au moins quelque chose pour avoir... enfin avoir une aide de l'assistante sociale pour qu'elle paye la dette locative, sinon je me retrouvais dehors. »

(Aïcha [E19], 25 ans, bac pro comptabilité)

De plus, une partie importante d'entre eux expliquent que l'allocation représente une aide pour la famille : une partie conséquente de ce qu'ils perçoivent contribue donc par solidarité aux dépenses familiales. Ainsi, Maëla [E1] déclare reverser la quasi-totalité de son allocation à ses parents bénéficiaires du RSA.

\section{Des parcours hyper-institutionnalisés}

L'accumulation des difficultés scolaires et de la précarité économique entraîne de nombreuses prises en charge par des assistantes sociales, par des conseillers d'orientation ou encore par des professionnels des services de protection de l'enfance. Les récits des jeunes sont marqués par le sentiment qu'au fil des années, ils « sont passés » d'une institution à une autre, d'un dispositif à un autre. Par exemple, depuis son enfance, Amidou [E20], 20 ans, niveau CAP, est suivi par un éducateur de prévention spécialisée ; William [E28], 22 ans, sans diplôme, a été en foyers puis en familles d'accueil depuis le divorce de ses parents ; quant à Maëla [E1], 19 ans, sans diplôme, elle a vécu en foyer de protection de l'enfance pendant deux ans.

À la prise en charge de problématiques familiales s'ajoute pour certains une prise en charge psychiatrique, suite à un diagnostic réalisé au sein de l'Éducation nationale ou par des centres médico-psychologiques. Rencontrant des problèmes de 
santé mentale ou de retard psychologique, ils ont eu un suivi psychologique au sein de foyers de protection de l'enfance et dans le système scolaire. Les entretiens ont aussi été l'occasion pour les jeunes de se livrer sur les troubles psychiques dont ils sont atteints. Schizophrénie, bipolarité ou encore agoraphobie ont pu donner lieu à des séjours en hôpital de jour voire à des périodes d'internement en institution psychiatrique. Pour ces types de pathologie, les enquêtés expriment une lassitude profonde vis-à-vis des institutions dans lesquelles ils sont passés.

«Ça fait 19 ans que je suis suivie par l'ASE ${ }^{6}$ j'en peux plus [...]. Maintenant l'ASE ça sert plus à rien [...]. Sans l'ASE, j'aurais peut-être pas avancé... Mais j'en peux plus. J'suis surveillée 24 heures sur 24 ... Je vais pas appeler quand je vais faire pipi. »

(Marion [E6], 19 ans, bac technologique)

\begin{abstract}
«[À propos de ses internements en hôpital psychiatrique] Ah c'est l'enfer hein, j'aurais préféré aller en prison comme je disais là-bas. [...] Ah c'est... c'est bizarre hein. On n'est jamais nous-même. Déjà on n'est jamais nous-même. Ici par exemple je vous parle, là-bas je pourrais pas vous parler comme ça hein. Je vous aurais limite même pas parlé [...]. Ben parce que ils nous donnent des médicaments qui font qu'on est ramolli. Et puis voilà. [...] Ce qu'il y a de plus difficile là-bas c'est... C'est l'isolement. Parce que j'ai fait beaucoup d'isolement moi là-bas, et c'est difficile hein, c'est ce qu'il y a de plus difficile à supporter. [...] Ben en fait, ben, c'est comme le mitard en prison, c'est une prison dans l'hôpital, t'es enfermé, tu vois personne. $[\ldots]$ Personne, à part quand ils nous amènent le repas, c'est le seul temps où je vois des gens, sinon je vois personne. Quand il faut prendre sa douche ou quoi, sinon je vois personne. »
\end{abstract}

(Selim [E53], 23 ans, CAP)

Ainsi, les caractéristiques socio-démographiques des « jeunes Garantie jeunes » révèlent des situations qui ne se limitent pas à une position sociale incarnée par les trois « ni ». Certes, ces jeunes correspondent bien aux attendus de la Garantie jeunes, au sens où ils ne sont ni en emploi, ni en formation, ni en stage et cumulent des situations de vulnérabilité économique. Toutefois, l'hyper-institutionnalisation de leur parcours, fait de ruptures diverses, invite à penser moins par la négative, comme le suppose la catégorie « Neets vulnérables », qu’en revenant sur la réalité de leur expérience sociale.

6. Aide sociale à l'enfance. 


\section{ENCADRÉ 2}

\section{L'hyper-institutionnalisation dans la relation d'enquête}

L'hyper-institutionnalisation s'est particulièrement observée dans la relation d'enquête, c'est-à-dire dans la manière dont les jeunes nous ont perçus et ont reçu les conditions d'entretien. Au cours de notre première rencontre, nous avons pris un ensemble de précautions pour nous présenter et justifier notre présence régulière auprès d'eux sur une période de plusieurs mois. D'emblée, nous nous sommes présentés en tant que « sociologues », c'est-à-dire comme des professionnels qui tentent de comprendre la société et le fonctionnement des groupes d'individus. Nous avons beaucoup insisté sur l'anonymat et la confidentialité des propos échangés, en précisant très clairement que nous ne ferions pas de retour aux conseillers ni à la direction de la mission locale afin qu'ils se sentent libres de critiquer le dispositif s'ils le jugeaient nécessaire. Malgré ces précautions, notre rôle n'a pas été complètement clair pour certains d'entre eux. Nous avons été souvent associés à des « intervenants sociaux » ou à des « policiers ».

Associer le sociologue à un intervenant social n'est pas anodin et est révélateur du parcours institutionnel des jeunes rencontrés ainsi que de leur rapport au dispositif. En effet, une partie d'entre eux ont confié qu'ils avaient l'habitude de se raconter, que ce soit auprès de conseillers de missions locales, de psychologues, d'assistantes sociales ou d'éducateurs spécialisés. Si certains ont montré des difficultés à s'exprimer dans les conditions imposées par l'entretien classique, d'autres au contraire ont fait preuve d'une certaine « aisance » à exposer leurs problèmes personnels. Cette injonction à raconter, déjà décrite en sociologie (AstiER, Duvoux, 2006), a eu pour conséquence un « déversement » de la part de certains jeunes. Ils se sont confiés sans retenue sur leur intimité, sur les drames et sur les épreuves émotionnelles auxquelles ils ont été confrontés (viols et agressions sexuelles, maltraitances physiques et psychiques, avortements, suicide d'un parent, maladies de longue durée d'un proche, troubles psychiques, internements, addictions). Si une partie des enquêtés nous a associés à des intervenants sociaux, une autre nous a spontanément comparés à des «policiers », à deux types de policier plus particulièrement : « celui qui accuse » et « celui qui protège ». L'image du « policier qui accuse » a été reprise par une poignée de jeunes, impliqués notamment dans le trafic de cannabis ou d'objets volés. Ces derniers, habitués aux interrogatoires de police vécus au cours de gardes à vue, ont manifesté une certaine méfiance à notre égard après avoir évoqué leurs activités illégales, en particulier lorsque nous les avons contactés par téléphone quelques mois après les avoir rencontrés en entretien. Ils craignaient d' " être sur écoute » et se sont davantage censurés quant à leurs pratiques illégales. Tout au long de notre enquête, nous nous sommes donc efforcés de les rassurer sur nos intentions en rappelant l'anonymat et la confidentialité des entretiens. Quant à l'image du « policier qui protège », elle a été reprise par un jeune homme, victime de viols pendant plusieurs années. Le récit de ces agressions sexuelles par son beau-père a été enregistré pendant un entretien à la brigade des mineurs. Ce jeune a spontanément comparé l'entretien sociologique (enregistré) à l'audition policière. Face à ces réactions, révélatrices de l'expérience sociale des jeunes rencontrés, nous avons ajusté notre discours de présentation à leur condition sociale et nous avons d'abord défini le métier de sociologue par ce qu'il n'est pas, c'est-à-dire un éducateur, une assistante sociale, un psychologue ou encore un policier. 


\title{
Ce que ne dit pas la catégorie « Neets vulnérables »
}

La définition de « Neets vulnérables » par la négative - «ni en emploi, ni en cycles d'études, ni en stage »-réduit ces jeunes à ce qu'ils ne sont pas et occulte leurs expériences de travail et une partie de leurs propriétés sociales.

\section{Des jeunes qui se sont déjà confrontés au monde du travail}

L'un des concepteurs de la Garantie jeunes rencontré considère que les jeunes Garantie jeunes sont « sans aucune expérience professionnelle », « des jeunes qui n'ont rien fait ». Les résultats de notre enquête viennent discuter ses propos. Ils confirment, d'une part, que le travail demeure une valeur centrale pour ces jeunes issus des classes populaires (MÉDA, VendRAmin, 2010). D'autre part, ils montrent que la plupart des enquêtés ont travaillé - de manière discontinue - et ont accédé à différents statuts d'emploi précaires (CDD, mission d'intérim). Au moment où ils entrent dans la Garantie jeunes, nombre d'entre eux ont donc vécu la discontinuité et la précarité de l'emploi, ce qui constitue une expérience professionnelle en soi, pourtant ignorée par les concepteurs de la Garantie jeunes. Ce constat est partagé par un ensemble de conseillers d'insertion ayant participé à la mise en œuvre du dispositif, comme le montre l'extrait d'entretien suivant :

\begin{abstract}
« De toute façon la notion de Neets, c'est de l'utopie, t'es Neet quand tu sors de troisième à la limite, mais sinon tu l'es plus. Ils ont tous - et heureusement d'ailleurs ils ont tous forcément eu des expériences, que ce soit de l'intérim, des stages, des choses comme ça. Donc à ce moment-là, ils sont plus Neets dans le sens théorique du terme. »
\end{abstract}

(Conseiller Garantie jeunes, chargé de l'accompagnement collectif [ML9])

Une faible partie des jeunes rencontrés au cours de l'enquête n'a jamais travaillé ou effectué un stage. On observe de rares cas d'inertie, c'est-à-dire des situations de jeunes passifs et démotivés. Ces derniers expliquent ne pas avoir été dans la capacité de rechercher du travail à cause de traumatismes tels que la maladie physique ou mentale, ou des violences subies, notamment sexuelles (COURONNÉ, LOISON-LERUSTE, SARFATI, 2016).

Les expériences de travail des jeunes ont eu lieu dans le cadre de stages en lien avec leur scolarité et avec d'autres programmes institués ou dispositifs d'insertion comme les $\mathrm{CAQ}^{7}$. Certains ont accumulé des expériences de stages, plus ou moins courtes, s'étalant sur plusieurs semaines ou sur plusieurs années. Rebecca [E23], suite au décès de son père, interrompt ses études et perd son « statut scolaire » à l'âge de 17

7. Le Contrat d'accès à la qualification (CAQ) est financé par le conseil régional et destiné aux jeunes demandeurs d'emploi âgés de 16 à 25 ans. Il permet d'obtenir une qualification de niveau V (CAP-BEP) et de niveau IV (baccalauréat). 
ans (BEAUd, Pialoux, 2003, p. 33). Elle commence alors une « carrière de stagiaire » (ibid., p. 35) pendant ses études et enchaîne une dizaine de stages dans différentes enseignes de supermarché (Couronné, 2017).

Pour d'autres, les expériences de stage se cumulent avec des missions d'intérim ou des contrats à durée déterminée, allant de quelques jours à plusieurs mois. À ces expériences, s'ajoute, pour bon nombre d'entre eux, du travail non déclaré. Ces jeunes ont participé à des déménagements, ont réalisé des « extras » dans la restauration ou ont participé à l'activité d'un père travaillant à son compte dans le secteur du bâtiment. D'autres ont travaillé le week-end dans des boîtes de nuit.

Si notre enquête de terrain a fait tomber l'idée d'une jeunesse qui n'aurait « jamais travaillé », elle met également en lumière l'expérience de recherche d'emploi des « jeunes Garantie jeunes » et les nombreux échecs qui en découlent. Ces jeunes sont apparus à bien des égards impliqués dans leurs recherches d'emploi. Le fait de proposer des ateliers « $\mathrm{CV}$ » et « lettres de motivation » pendant la phase inaugurale du dispositif incite à penser que les jeunes seraient complètement ignorants des règles et des pratiques de recherche d'emploi, comme contacter les entreprises, consulter les annonces sur différents sites internet, s'inscrire dans une agence d'intérim, se déplacer pour déposer des candidatures spontanées. Une fois de plus, les résultats de notre enquête permettent de nuancer les représentations sociales de la jeunesse édifiées par les concepteurs de la Garantie jeunes. Ainsi, David [E2] déclare avoir envoyé soixante-quatre lettres de motivation à des entreprises. Michaela [E27] raconte quant à elle s'assigner quotidiennement à cette tâche et organiser de manière disciplinée son temps consacré à la recherche d'emploi :

«Comment ça se passe chez vous quand vous faites les recherches sérieusement [comme vous dites] ?

- Ben je vais sur Pôle emploi, sur le Bon coin, je passe dans les magasins, c'est bête mais je vais dans les petits magasins, comme $\mathrm{P}^{* * * *}$ et $\mathrm{M}^{* * * *}, \mathrm{j}$ ' ai vu qu'ils cherchaient, donc j'ai noté, et après quand je rentre chez moi ben je fais ma lettre et je vais la déposer.

- D'accord.

- Et je sais aussi qu' il y a $\mathrm{P}^{* * * * *}$ aussi qui recherche, pareil. Donc je sais que je vais... Y'a aussi $\mathrm{O}^{* * *}$ qui cherche, donc je vais y aller. C'est bête, on pourrait croire que je fais du shopping, mais en fait je recherche les affiches qui recherchent.

- D'accord. Et alors ça a donné des choses ou pas ?

- Ben là j'attends. C'est pareil, y'a un nouveau magasin qui va ouvrir, c'est de la marque, j'irai faire un tour. Y'a au moins six réponses que je dois attendre. Donc oui... et après faut s'y prendre sérieusement, faut pas avoir peur de bouger. [...]

- Et du coup, à la mission locale, quand est-ce que vous y retournez ?

- On a des rendez-vous une fois par semaine, en fait. Moi j'ai un peu moins de rendez-vous parce que vu que je me débrouille dans mes recherches, qu'ils savent que je suis autonome, j'y vais quand j'ai besoin. Ils me donnent quand même des rendez-vous, mais si j'ai pas besoin... 
- Du coup là vous faites quoi de vos journées?

- Ben je recherche du travail. Je suis là-dessus pour l'instant.

- Mais vous passez huit heures à chercher du travail ?

- Ben oui mais sinon des fois je sors, ouais, je... de toute façon on est payés pour faire ça, au moins ça m'occupe. Puis sur l'ANPE les annonces, elles sont pas mises, moi je regarde mes mails le matin, après midi et à 5 heures, parce que les offres les plus intéressantes, elles viennent après 17 heures. J'en ai vu plusieurs comme ça, je me suis faite avoir, le lendemain. »

(Michaela [E27], 21 ans, sans diplôme)

Une partie des jeunes, au moment où ils intègrent la Garantie jeunes, se sont déjà confrontés au monde du travail et à la « loi du marché ». Du fait de leur faible qualification, ils ont accédé à une série d'emplois précaires, aux conditions de travail éprouvantes (travail répétitif, horaires nocturnes). Ils ont été mis à rude épreuve, que ce soit en affrontant le jugement des employeurs à leur égard ou l'absence de réponse à leurs candidatures spontanées. Les employeurs ne cessent de leur renvoyer leur manque d'expérience comme l'explique Jordan [E39], et bien d'autres encore :

«Là, je cherche du travail en plomberie. J'ai des diplômes, mais on me dit que non, parce que j'ai pas d'expérience. Vu que j'étais en lycée pro, j'ai pas du tout d'expérience : j'ai qu'une semaine par-ci, une semaine par-là, avec les intérim [...] je les appelle [les agences d'intérim] et je leur dis que je suis toujours dispo... Mais là je leur ai bien fait comprendre que là, il me faut des missions un peu longues, quand même, parce que... créer un peu d'expérience. Mais bon après faut les trouver, des patrons qui veulent des débutants, c'est rare. »

(Jordan [E39], 22 ans, deux CAP)

Les réticences des patrons se manifestent également par les refus que les jeunes ont essuyés à l'occasion de demandes de contrat d'apprentissage.

La manière dont les « jeunes Garantie jeunes » vivent et se représentent le travail s'inscrit aussi dans l'héritage d'une condition familiale marquée par la précarité et la discontinuité.

\section{La précarité et la discontinuité : une expérience familiale}

Ces parcours de jeunes sont en partie déterminés par l'expérience professionnelle et la condition sociale de leurs parents ainsi que celles de leurs frères et sœurs. Leurs parents appartiennent pour la totalité d'entre eux aux franges les plus démunies des classes populaires. En effet, les classes populaires peuvent se définir selon trois caractéristiques : «petitesse du statut professionnel ou social, étroitesse des ressources économiques - sans que cela signifie nécessairement précarité - éloignement par 
rapport au capital culturel, et d'abord par rapport à l'école ${ }^{8}$ » (SCHWARTZ, 2011). Les parents des « jeunes Garantie jeunes » exercent des emplois précaires relevant de l'aide à la personne, de la manutention, de l'entretien et du bâtiment. Beaucoup d'entre eux sont sans emploi, certains sont malades ou en situation de handicap. Leurs revenus sont faibles, nombreux sont ceux qui touchent le RSA ${ }^{9}$ et bénéficient d'aides sociales (APL ${ }^{10}$, bons alimentaires, aides pour l'électricité).

Le père de Kevin [E3], anciennement maraîcher, est sans emploi depuis plusieurs années. Âgé de 51 ans, « il galère un peu pour retrouver du travail ». Quant à sa mère, âgée de 50 ans, elle est aujourd'hui mère au foyer après avoir été couturière. Au cours d'un entretien, Kevin s'exprime en ces termes :

«Et alors tous, (tes frères et sœurs) ont travaillé... donc le grand qui est coffreur, maintenant il est en CDI, il est responsable. Les autres ils ont quoi, comme type de... contrats, vous savez?

- Ma sœur qui est caissière, elle a un CDI. Mon autre sœur c'est pareil, c'est un CDI mais elle c'est du mi-temps. Après ben ma sour qui travaille pas, c'est du chômage, du CDI chômage [rire]. Et mon petit frère, c'est des contrats d'apprentissage. »

(Kevin [E3], 21 ans, deux CAP)

En ce qui concerne Maëla [E1], ses parents perçoivent le RSA et sont sans emploi depuis plusieurs années. Le père de Maëla était employé polyvalent en supermarché et sa mère était caissière. Les deux frères de Maëla (21 et 23 ans) sont également sans emploi : « [À la maison], ben ils font rien [...]. Y'a des disputes des fois mais ça va... [...] Chaque fois il y a des problèmes d'argent et tout ça... » Les revenus familiaux sont donc très faibles (800 euros par mois) : «Les factures payées, il reste même pas 200 euros. »

Quant à Rebecca [E23], elle est la cadette d'une fratrie constituée d'un frère aîné (âgé de 22 ans, préparateur de commandes en usine) et d'une sœur benjamine (âgée de 17 ans, en CAP employé de commerce). Sa mère est sans emploi, après avoir été femme de ménage. Le père de Rebecca, ancien brancardier, est décédé alors qu'elle était âgée de 18 ans. Cette jeune femme déclare ne pas avoir de rêves. Travailler à l'usine représente « un choix du destin, mais un choix forcé, produit par des conditions d'existence

8. Un nombre important d'enquêtés portent des prénoms d'origine immigrée. Nous avons choisi dans le processus d'anonymisation de conserver l'origine sociale des prénoms de nos enquêtés pour rendre compte du fait qu'une partie d'entre eux sont des descendants d'immigrés, tandis que d'autres ont eux-mêmes connu une migration. Toutefois, nous ne consacrons pas de développement spécifique sur ce point dans l'article dans la mesure où nous nous concentrons sur les effets du dispositif sur les parcours des « jeunes Garantie jeunes ». Or, rien dans l'enquête ne nous permet d'affirmer que les professionnels agiraient d'une manière différente avec les migrants ou descendants d'immigrés. Dans le cas présent, on peut souligner qu'en tant qu'immigrés ou descendants d'immigrés, ils appartiennent, comme c'est le cas le plus fréquent, aux classes populaires (SiBLOT et al., 2015).

9. Pour pouvoir bénéficier de la Garantie Jeunes, il faut que les ressources du jeune ne dépassent pas 524,68 euros. Toutefois, à titre exceptionnel, des jeunes dont les ressources dépassent ce plafond peuvent bénéficier de la Garantie jeunes si leur situation le justifie. URL : https://www.service-public.fr (pages consultées le 11 mai 2016).

10. Aide personnalisée au logement. 
qui, en excluant comme pure rêverie tout autre possible, ne laissent d'autre choix que le goût du nécessaire » (BourdiEu, 1979, p. 173). Dès son entrée à la Garantie jeunes, Marion [E6] souhaite devenir gardien de la paix, mais les échecs consécutifs au concours et les « rappels au réalisme » des conseillers (ZuNIGO, 2010) l'obligent à faire évoluer ses aspirations dans la mesure où elle accepte " des boulots ingrats ", «j'ai pas le choix ». Désormais connue des agences d'intérim, elle enchaîne plusieurs missions pendant six mois : préparation de commandes, vente en libre-service, mise sous plis, travail à la chaîne dans une usine de parfum.

Cette attitude renvoie à ce que Xavier ZuniGo appelle le « deuil des grands métiers » (ibid.). En se confrontant au monde du travail, certains jeunes intériorisent «le sens social » ou le « sens de la place » (ZARCA, 1999), ils intériorisent les possibilités qui leur sont offertes compte tenu de leur qualification. De la même manière, Julie [E37] se dit prête à travailler quel que soit le poste : «Aujourd'hui, on choisit plus son travail. »

Ces parcours se caractérisent par un "processus d'ouverture/fermeture » de l'espace des possibles. Si, dans un premier temps, certains aspiraient à des postes socialement valorisés comme des emplois de fonctionnaires, tels que professeur, gendarme ou infirmière, au fur et à mesure des mois, ils ont revu leurs attentes à la baisse. Qu'il s'agisse de Kevin [E3], de Rebecca [E23], de Marion [E6] ou encore de Julie [E37], ces jeunes sont représentatifs d'une frange de la jeunesse issue des classes populaires les plus démunies, consciente de sa « place », de sa condition sociale et de la petitesse de son niveau scolaire ; une jeunesse ayant le « sens des limites » (BOURDIEU, 1984). Confrontés au chômage de masse, ils ont intériorisé l'incertitude du marché du travail comme norme sociale (PAUgAM, 2007 ; SARFATI, 2012). Cette expérience de la précarité, acquise par leur expérience personnelle, mais également à travers celles de leurs parents et de leurs frères et sœurs, conditionne leur rapport au travail et à l'emploi, ainsi qu'aux dispositifs d'insertion de manière plus générale.

Au regard de ce constat, ce dispositif apparait donc moins comme la garantie d'une première expérience professionnelle que comme la garantie d'un accompagnement à «tenir au travail », à « faire face », à accepter «sa place». Cet accompagnement se joue plus particulièrement dans la relation des jeunes avec les conseillers.

\section{Parcours de jeunes sous l'effet de l'accompagnement}

Parce que travailler sur autrui suppose de construire une relation (DUBET, 2002), les conseillers d'insertion des missions locales n'ignorent pas le passé professionnel des jeunes. Dès les premiers moments de l'accompagnement collectif, les curriculum vitæ sont remis à jour et retravaillés par les conseillers de la Garantie jeunes, plus particulièrement dans la mission locale verte. Une conseillère « relations entreprises » consacre une partie de ses activités à cette tâche : 
« Je suis dans la salle d'accueil de la mission locale où se déroulent simultanément plusieurs scènes. Sur le côté devant des postes informatiques, cinq jeunes recherchent des vidéos sur YouTube, présentant des films institutionnels comme ceux réalisés par Pôle emploi. Ces vidéos servent à présenter différents métiers à des candidats éventuels (cariste, conductrice de bus, magasinier, etc.). Une ambiance bon enfant se fait jour devant les écrans. Les jeunes rient, se moquent gentiment des vidéos, s'imaginent dans tel ou tel métier, se chambrent les uns les autres. De temps en temps, profitant de l'absence de Camille [ML8], la conseillère, qui fait des allers-retours dans son bureau, les jeunes regardent des pages internet personnelles sur Facebook ou surfent sur des sites de football, etc. Détendue, l'ambiance est globalement studieuse. À l'autre bout de la pièce, autour de la table centrale, d'autres jeunes relisent leurs lettres de motivation et leurs curriculum vitæ. Camille échange avec eux à tour de rôle et des discussions s'engagent entre les jeunes autour de la table et ceux devant les écrans. Concrètement, Camille est en train d'animer un groupe de jeunes qui s'interrogent sur les métiers qu'ils pourraient faire, relit, corrige des CV et explique aux jeunes comment mieux présenter leurs parcours, et s'interrompt pour s'isoler avec un jeune ayant manifestement une question trop personnelle pour être évoquée en public. »

(Extrait de notes ethnographiques)

En partant de l'analyse de deux curriculum vitæ (figures 1 et 2), nous sommes en mesure de documenter finement la manière dont ces « jeunes Garantie jeunes » cheminent dans le dispositif. En articulant la réalité du parcours et l'accompagnement, le sociologue se donne la possibilité de comprendre comment les destinataires s'approprient le dispositif d'action publique. Le parcours de Maëla [E1] est révélateur d'un processus de fermeture de l'espace des possibles, alors que celui de Kevin [E3] symbolise le processus inverse, une ouverture de l'espace des possibles.

Après l'interruption de ses études à l'âge de 14 ans, Maëla s'inscrit à la mission locale et obtient un CAQ en septembre 2014. Durant quatre mois, la jeune femme suit des cours de remise à niveau (en français notamment) et enchaîne plusieurs stages dans la vente puis en maison de retraite. En janvier 2015, sans emploi, elle reprend contact avec la mission locale pour obtenir un Civis :

« Je sais pas [ce que c'est] en fait. C'est mon frère qui m'en avait parlé, parce qu'en fait ils donnaient 70 euros, donc c'était quand même ça. »

Sa conseillère lui a appris l'existence de la Garantie jeunes :

«Elle sait que mes parents ont des RSA, que je touche pas beaucoup d'argent, $[\ldots]$ que je correspondais aux critères...»

(Maëla [E1], 19 ans, sans diplôme)

Contrairement au CAQ, la Garantie jeunes lui semble davantage tournée vers « le monde du travail ». Les conseillers, quant à eux, sont «plus à l'écoute», «viennent plus vers nous », « expliquent bien ». Ils sont aussi « autoritaires mais ils vont rigoler avec nous ». 
FIGURE 1 - Curriculum vitæ de Maëla [E1]

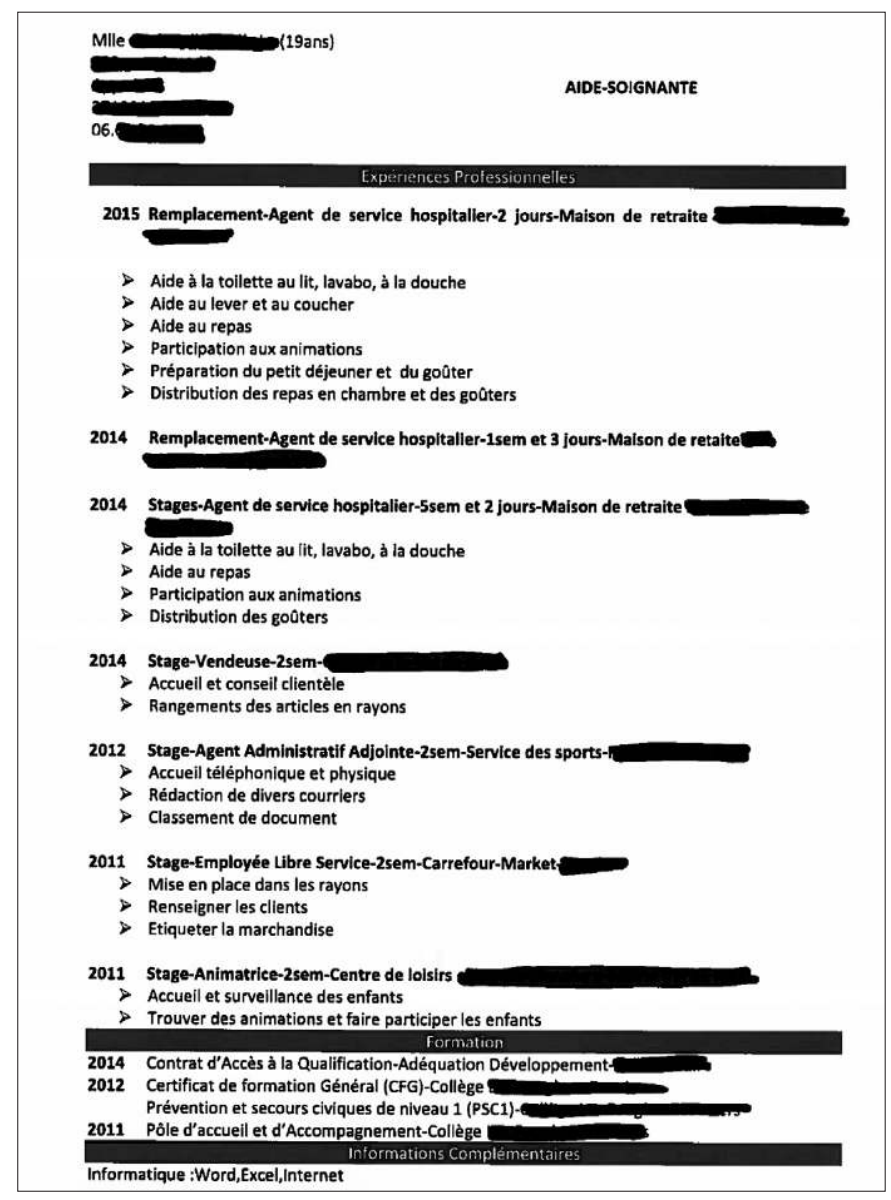

Respectant la consigne du work first et ayant intégré la philosophie du projet professionnel (ZuNIGO, 2010), les conseillers d'insertion invitent les jeunes - dès leur entrée dans le dispositif - à contacter d'anciens employeurs pour reconduire leur CDD. C'est le cas de Maëla. Quand elle entre à la Garantie jeunes, elle a travaillé ponctuellement dans une maison de retraite médicalisée en tant qu'auxiliaire de vie. Incitée par les conseillers de la Garantie jeunes à « rentabiliser » cette expérience, elle reprend contact avec la direction et enchaîne de manière plus intensive les remplacements pendant un an. Maëla espère pouvoir décrocher un contrat de longue durée, «quelque chose de plus stable ». Au mieux, elle obtient un contrat de deux mois pendant la période estivale. Devant la difficulté des tâches à effectuer (aide à la toilette de personnes âgées) et la précarité de l'emploi (enchaînement de CDD de courte durée), Maëla se décourage. Elle déclare alors au cours du dernier entretien : 
« Et dans ce que tu faisais au niveau du travail auprès des personnes âgées, tu faisais quoi exactement?

- Ben, je faisais tout ce que fait une aide-soignante en fait. Je faisais de l'accompagnement pour les toilettes, tout, pour l'aide aux repas...

- Et ça, ça t'a... Tu disais que t'avais perdu patience, par rapport à tout ça...

- Ben oui, en fait, d'enchaîner plusieurs mois d'affilée, avec la fatigue et tout, au bout d'un moment, tu perds patience...

- En fait, t'as réalisé que...

- Ben, c'était dur, quand même... [...] Vu que je faisais des petits contrats à chaque fois, je voyais pas que... Sur plusieurs mois, dans la durée, ben, c'était dur, quoi.

- Ouais. Et qu'est-ce qui est dur pour toi ? C'est la fatigue tu m'as dit...

- Ouais, c'est la fatigue, et puis même, je sais pas comment dire ça, les personnes âgées, c'est dur quand même... La patience... faut tout, quoi. »

(Maëla [E1], 19 ans, sans diplôme)

À l'initiative des conseillers d'insertion de la Garantie jeunes, elle entreprend un Service civique à partir de septembre 2015 dans une école primaire. Elle y effectue des activités de secrétariat auprès de la direction et accompagne les enfants en sortie scolaire (20 heures par semaine). Alors que Maëla s'apprête à sortir du dispositif (après avoir été accompagnée pendant un an comme le prévoit la loi), la jeune femme se dit un peu « perdue » et déclare ne pas savoir « quoi faire après ». Loin d'avoir accédé à l'autonomie, Maëla manifeste un besoin persistant d'accompagnement au-delà de la période prévue.

Le cas de Kevin [E3] est tout autre. Son curriculum vitæ indique qu'il est titulaire de deux CAP. Il enchaîne des contrats de courte durée dans différents domaines. Il est successivement couvreur, chauffagiste, manutentionnaire, ce qui signale une certaine polyvalence. Kevin a également effectué des stages de longue durée (au minimum un an) pendant plusieurs années.

Dès son entrée à la Garantie jeunes, ce jeune homme semble enthousiasmé par le programme proposé : «SST » et l'habilitation électrique, « sur le curriculum vitæ, c'est bien »; l'atelier entretien d'embauche, «c'est vraiment pas mal pour apprendre à parler [aux patrons] ». Il a alors pour objectif de «trouver un emploi stable dans le chauffage ». Soutenu par les conseillers, il reprend contact avec les agences d'intérim dans lesquelles il est déjà inscrit (au moment où il intègre la Garantie jeunes), enchaînant ainsi des missions d'intérim : manutention, réparation de chambre froide. Au bout de quelques mois, il décroche un contrat de formation dans une entreprise multinationale et suit un baccalauréat professionnel « canalisation » :

«C'est positif pour toi la Garantie jeunes?

- Oui. Carrément. Ça m'a servi.

- Ça t'a servi à quoi ?

- Ben, déjà à rentrer chez **** [entreprise multinationale de services collectifs].

Enfin, je n'aurais jamais pensé à postuler chez ***... 
- Oui.

- Oui, ça m'a carrément aidé.

- Qu'est-ce que tu as appris, à la Garantie jeunes ?

- À ne pas désespérer.

- Oui.

- Oui. Parce que, à des moments c'est dur.

- Oui. Qu'est-ce qui est dur?

- Ben, quand tu n'as pas de travail et... Enfin, moi, je n'ai jamais été habitué à rester six mois sans rien faire. Ça c'est dur ça, pour moi. »

(Kevin [E3], 21 ans, deux CAP)

FIGURE 2 - Curriculum vitæ de Kévin [E3]

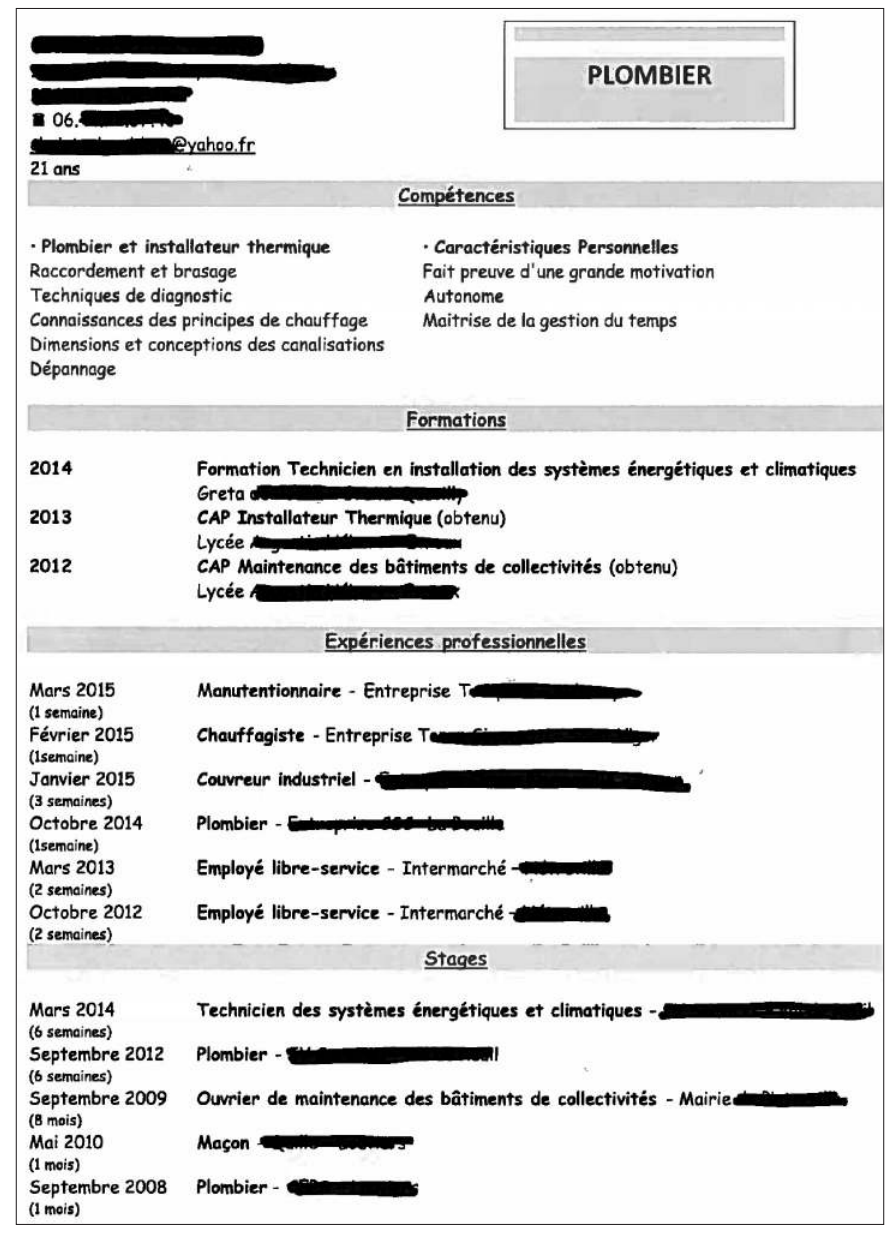


À l'issue de la Garantie jeunes, le jeune homme aspire à une position sociale plus élevée que celle qu'il occupe au moment de l'entretien. En effet, Kevin souhaite accéder à un poste de « chef », pour reprendre ses mots. Il insiste - comme beaucoup d'autres - sur la disponibilité des conseillers d'insertion et sur leur approche globale de la situation des jeunes ; « les conseillers ne sont pas là que pour le professionnel ». Kevin confie également que, sans la Garantie jeunes, « [il] serait parti loin, [il] aurait rejoint le noir » faisant référence à sa consommation d'alcool et de cannabis. Enfin, il conclut le dernier entretien en affirmant : « Je suis en train de m'en sortir comme il faut ». En reconstituant le parcours de Kevin, il apparaît très clairement que la proposition d'un baccalauréat professionnel par l'une des conseillères de la Garantie jeunes en alternance dans une grande entreprise française a contribué à ouvrir « l'espace des possibles » et à envisager l'avenir plus sereinement.

Ces deux cas montrent que les conseillers d'insertion ne réduisent pas les jeunes à la condition de Neets. Ils s'appuient sur leurs expériences antérieures pour construire un accompagnement adapté aux difficultés qu'ils rencontrent. Ainsi, nous avons pu observer des situations dans lesquelles les conseillers aident à résoudre, souvent dans l'urgence, des problèmes de différentes natures (familiale, de logement, de santé, etc.), sans renoncer à centrer principalement leur action sur l'emploi. La priorité est donnée à l'emploi et à la multiplication des expériences professionnelles. L'analyse de ces deux parcours rend bien compte de la manière dont les conseillers d'insertion traduisent la stratégie de work first dans leurs pratiques professionnelles. Sans ignorer les difficultés que les jeunes rencontrent sur différents pans de leur existence, les conseillers misent sur le travail pour donner des perspectives à ces jeunes. Ils se sont, en effet, peu emparés de la proposition de médiation active ${ }^{11}$. Il ne reste pas moins qu'une minorité de jeunes traverse des épreuves trop difficiles pour voir leur situation s'améliorer sous l'effet du dispositif (Couronné, Loison-Leruste, SARFATI, 2016). L'ancrage dans la vulnérabilité économique et sociale les empêche de se saisir de la Garantie jeunes et fait échouer la stratégie du work first, témoignant de la nécessité pour certains jeunes de résoudre des difficultés immédiates avant d'être en situation d'accéder à un travail et plus encore à un emploi.

En introduisant la catégorie « Neets vulnérables » comme cible d'un dispositif d'action publique, les concepteurs du dispositif ont réussi à rendre visibles les jeunes «ni en emploi, ni en formation, ni en stage » et ont permis à ces jeunes de trouver un accompagnement vers l'emploi qui les mobilise. La Garantie jeunes se présente comme une avancée sur le plan des droits des jeunes puisqu'elle apporte, à travers l'allocation et l'accompagnement collectif, des réponses à leurs difficultés financières et à leurs difficultés d'insertion sociale et professionnelle.

11. Voir l'article de Nicolas FARVAQue et Carole Tuchszirer (2018) dans ce dossier. 
La catégorie « Neets vulnérables » contribue donc à faire émerger un problème public en constituant ces jeunes comme la cible d'un dispositif. Si les concepteurs de la Garantie jeunes mobilisent une définition sociétale (BLUMER, 1971), fondée sur une vision par la négative de cette jeunesse, il est assez clair que dans les missions locales, les conseillers « font avec » l'épaisseur sociale des jeunes entrés dans le dispositif. On peut alors mettre en évidence l'existence de différents niveaux de catégorisation. Les concepteurs de politiques publiques raisonnent à leur échelle en termes de public cible à atteindre et adoptent une stratégie de résolution des problèmes (work first et médiation active). À l'échelle du travail concret d'accompagnement, le passé personnel et professionnel des personnes accompagnées est mobilisé, comme en atteste le fait que les conseillers n'emploient jamais l'expression « Neets vulnérables » pour désigner les jeunes qu'ils accompagnent.

Créée et instaurée par le haut, la catégorie est opératoire au sens où elle permet de faire entrer dans le dispositif des jeunes qui correspondent bien à la définition qu'elle propose. Délaissée par le bas, elle disparaît pour laisser la place à des situations de jeunesses que taisent les indicateurs statistiques. Le travail sur autrui suppose que les conseillers d'insertion se concentrent sur la relation qu'ils ont à construire avec les jeunes. Pour ce faire, ils s'appuient davantage sur ce que les jeunes sont, sur leurs atouts à faire valoir dans la recherche d'emploi, plutôt que sur le fait qu'ils soient « ni-ni-ni ».

\section{BIBLIOGRAPHIE}

Astier I., Duvoux N. (2006), La Société biographique : une injonction à vivre dignement, Paris, L'Harmattan, coll. « Logiques sociales ».

Beaud S., Pialoux M. (2003), Violences urbaines, violence sociale. Genèse des nouvelles classes dangereuses, Paris, Fayard.

Becquemin M., Mardon A., Couronné J. (2012), « Absentéismes scolaires. Étude des motifs exprimés par les jeunes dans le cadre du rappel à la loi sur l'obligation scolaire », Sociétés et jeunesses en difficulté, printemps, $\mathrm{n}^{\mathrm{0}} 13$; en ligne : https://sejed.revues.org, consulté le 22 juin 2017.

Bizeul D. (2007), « Que faire des expériences d'enquête ?Apports et fragilité de l'observation directe », Revue française de science politique, vol. 57, pp. 69-89.

Blumer H. (1971), “Social Problems as Collective Behavior.”, Social Problems, vol. 18, n³, traduit par Riot Laurent, « Les problèmes sociaux comme comportements collectifs », Politix, vol. 17, n 67, pp. 185-199, (2004).

Bourdieu P. (1979), La Distinction, critique sociale du jugement, Paris, Minuit.

BouRdiEu P. (1984), « Espace social et genèse de classe », Actes de la recherche en sciences sociales, $\mathrm{n}^{\circ} 52$, pp. 1-14.

CONSEIL DE L'UNION EUROPÉENNE (2013), Recommandation du conseil sur l'établissement d'une garantie pour la jeunesse, 22 avril ; en ligne : http://eur-lex.europa.eu, consulté le 3 juin 2017. 
CONSEIL EUROPÉEN (2012), Conclusions, 28 et 29 juin, en ligne : http://www.consilium.europa. eu/uedocs/cms_data/docs/pressdata/fr/ec/131408.pdf.

Couronné J., Loison-Leruste M., SARfati F. (2016), La Garantie jeunes en action. Usages $d u$ dispositif et parcours de jeunes, Rapport de recherche $\mathrm{n}^{\circ} 101$, Paris, Centre d'études de l'emploi et du travail.

Couronné J. (2016), « Pour un usage sociologique du dessin. Réflexion méthodologique à partir d'une étude de cas », Agora Débats/Jeunesses, n ${ }^{\circ}$ 74, pp. 25-38.

Couronné J. (2017), «"Peu importe, du moment que je travaille”. L'usine comme "goût de nécessité" », Nouvelle revue du travail, n ${ }^{\circ}$ 10, mis en ligne le 2 mai 2017 : http://nrt.revues. org, consulté le 16 juin 2017.

Desrosières A. (1993), La Politique des grands nombres. Histoire de la raison statistique, Paris, La Découverte.

Dubet F. (2002), Le Déclin de l'institution, Paris, Le Seuil.

EUROFOUnd (2012), NEETs - Young People not in Employment, Education or Training: Characteristics, Costs and Policy Responses in Europe, Luxembourg, Office des publications de l'Union européenne.

FARvaque N., Tuchszirer C., 2018, « Théorie et pratique de la Garantie jeunes dans les missions locales : travailler au changement, travailler dans le changement », Travail et Emploi, $\mathrm{n}^{\mathrm{o}} 153$, pp. 15-40.

FurLONG A. (2007), "The Zone of Precarity and Discourses of Vulnerability: Neet in the UK", The Journal of Social Sciences and Humanities, $\mathrm{n}^{\circ}$ 381, 2007, pp. 101-121.

Gautié J. (2015), Le Chômage, Paris, La Découverte.

Gusfield J. (2009), La Culture des problèmes publics. L'alcool au volant : la production d'un ordre symbolique [éd. originale, The Culture of Public Problems: Drinking-Driving and the Symbolic Order, Chicago, The University of Chicago Press, 1981], Paris, Economica.

Istance D., Rees G., Williamson H. (1994), Young People not in Education, Training or Employment in South Glamorgan, South Glamorgan Training and Enterprise Council, Cardiff.

Lepoutre D. (1997), Coeur de banlieue. Codes, rites et langages, Paris, Odile Jacob.

MÉdA D., VENDRAMIN P. (2010), « Les générations entretiennent-elles un rapport différent au travail ?», SociologieS [en ligne], Théories et recherches, mis en ligne le 27 décembre 2010 : http://sociologies.revues.org/3349, consulté le 19 juin 2017.

Paugam S. (2007), Le Salarié de la précarité : les nouvelles formes de l'intégration [2000], Paris, Presses universitaires de France.

SARFATI F. (2012), Du côté des vainqueurs. Une sociologie de l'incertitude sur les marchés du travail, Villeneuve d'Ascq, Presses universitaires du Septentrion.

Siblot Y., Cartier M., Coutant I., Masclet O., Renahy N. (2015), Sociologie des classes populaires contemporaines, Paris, Armand Colin. 
Schwartz O. (2011), « Peut-on parler des classes populaires ? », La Vie des idées ; en ligne : https://journals.openedition.org/lectures/6326, consulté le 15 février 2016.

Wargon E., Gurgand M. (2013), Garantie jeunes, Synthèse des travaux du groupe, mai.

Weber F. (2009), Manuel de l'ethnographe, Paris, Presses universitaires de France.

ZARCA B. (1999), «Le sens social des enfants », Sociétés contemporaines, vol. 36, $\mathrm{n}^{\circ} 1$, pp. 67-101.

Zunigo X. (2010), « Le deuil des grands métiers. Projet professionnel et renforcement du sens des limites dans les institutions d'insertion », Actes de la Recherche en sciences sociales, $\mathrm{n}^{\mathrm{o}} 184$, pp. 58-71. 


\section{ANNEXE - TABLEAU RÉCAPITULATIF DES CARACTÉRISTIQUES SOCIALES ET DES ENTRETIENS MENÉS AUPRÈS DES ENQUÊTÉS}

\begin{tabular}{|c|c|c|c|c|c|c|c|}
\hline Code & Pseudo & Statut/Fonction & Sexe & Âge & Niveau d'étude & $\begin{array}{l}\text { Nombre } \\
\text { d'entretiens } \\
\text { réalisés }\end{array}$ & Autre \\
\hline E1 & Maëlla & Destinataire Garantie jeunes & $\mathrm{F}$ & 19 & sans diplôme & 2 entretiens & Focus group \\
\hline E2 & David & Destinataire Garantie jeunes & $\mathrm{H}$ & 18 & $\begin{array}{l}\text { sans diplôme, } \\
\text { niveau CAP ébénisterie }\end{array}$ & 2 entretiens & \\
\hline E3 & Kévin & Destinataire Garantie jeunes & $\mathrm{H}$ & 21 & $2 \mathrm{CAP}$ & 2 entretiens & \\
\hline E4 & Nicolas & Destinataire Garantie jeunes & $\mathrm{H}$ & 25 & CAP & 2 entretiens & \\
\hline E5 & Laura & Destinataire Garantie jeunes & $\mathrm{F}$ & 20 & niveau CAP & 2 entretiens & \\
\hline E6 & Marion & Destinataire Garantie jeunes & $\mathrm{F}$ & 19 & bac tech & 2 entretiens & Focus group \\
\hline E7 & Johnny & Destinataire Garantie jeunes & $\mathrm{H}$ & 18 & niveau CAP & 2 entretiens & \\
\hline E8 & Gladys & Destinataire Garantie jeunes & $\mathrm{F}$ & 18 & brevet des collèges & 1 entretien & \\
\hline E9 & Tony & Destinataire Garantie jeunes & $\mathrm{H}$ & 21 & $\begin{array}{l}\text { brevet des collèges, } \\
\text { niveau bac pro electrotech }\end{array}$ & 2 entretiens & Focus group \\
\hline E10 & Steeve & Destinataire Garantie jeunes & $\mathrm{H}$ & 24 & bac pro commerce & 2 entretiens & \\
\hline E11 & Foussenou & Destinataire Garantie jeunes & $\mathrm{H}$ & 24 & $\begin{array}{l}\text { CAP agent polyvalent } \\
\text { de restauration }\end{array}$ & 2 entretiens & \\
\hline E12 & Cédric & Destinataire Garantie jeunes & $\mathrm{H}$ & 18 & sans diplôme & 1 entretien & \\
\hline E13 & Angélina & Destinataire Garantie jeunes & $\mathrm{F}$ & 22 & BEPC & 2 entretiens & \\
\hline E14 & Julien & Destinataire Garantie jeunes & $\mathrm{H}$ & 21 & $\begin{array}{l}\text { CAP mécanique } \\
\text { automobile }\end{array}$ & 1 entretien & \\
\hline E15 & Yasmine & Destinataire Garantie jeunes & $\mathrm{F}$ & 21 & brevet des collèges & 2 entretiens & \\
\hline E16 & Neil & Destinataire Garantie jeunes & $\mathrm{H}$ & 19 & $\begin{array}{l}\text { Segpa } \\
\text { niveau CAP cuisine }\end{array}$ & 2 entretiens & \\
\hline E17 & Wilfried & Destinataire Garantie jeunes & $\mathrm{H}$ & 21 & sans diplôme & 2 entretiens & Focus group \\
\hline E18 & Thomas & Destinataire Garantie jeunes & $\mathrm{H}$ & 23 & sans diplôme & 2 entretiens & \\
\hline E19 & Aicha & Destinataire Garantie jeunes & $\mathrm{F}$ & 25 & bac pro comptabilité & 1 entretien & \\
\hline E20 & Amidou & Destinataire Garantie jeunes & $\mathrm{H}$ & 20 & $\begin{array}{l}\text { sans diplôme, } \\
\text { niveau CAP ébénisterie }\end{array}$ & 1 entretien & \\
\hline E21 & Kader & Destinataire Garantie jeunes & $\mathrm{H}$ & 20 & niveau CAP & 2 entretiens & \\
\hline E22 & Oliver & Destinataire Garantie jeunes & $\mathrm{H}$ & 21 & niveau BTS & 2 entretiens & \\
\hline E23 & Rebecca & Destinataire Garantie jeunes & $\mathrm{F}$ & 21 & $\begin{array}{l}\text { CAP employé de } \\
\text { commerce multi-spécialités }\end{array}$ & 2 entretiens & Focus group \\
\hline E24 & Nourredine & Destinataire Garantie jeunes & $\mathrm{H}$ & 24 & $\begin{array}{l}\text { sans diplôme, niveau CAP } \\
\text { serrurerie-métallerie }\end{array}$ & 2 entretiens & \\
\hline E25 & Asma & Destinataire Garantie jeunes & $\mathrm{F}$ & 24 & sans diplôme & 2 entretiens & Focus group \\
\hline E26 & Clémence & Destinataire Garantie jeunes & $\mathrm{F}$ & 25 & BEP vente & 1 entretien & Focus group \\
\hline E27 & Michaela & Destinataire Garantie jeunes & $\mathrm{F}$ & 21 & sans diplôme & 2 entretiens & \\
\hline E28 & William & Destinataire Garantie jeunes & $\mathrm{H}$ & 22 & collège & 2 entretiens & \\
\hline E29 & Fouaad & Destinataire Garantie jeunes & $\mathrm{H}$ & 19 & niveau BEPC & 2 entretiens & \\
\hline E30 & Mathieu & Destinataire Garantie jeunes & $\mathrm{H}$ & 23 & CAP & 1 entretien & \\
\hline E31 & Naïm & Destinataire Garantie jeunes & $\mathrm{H}$ & 20 & sans diplôme, arrêt collège & 1 entretien & \\
\hline E32 & Anne & Destinataire Garantie jeunes & $\mathrm{F}$ & 21 & sans diplôme, arrêt collège & 2 entretiens & Focus group \\
\hline E33 & Elodie & Destinataire Garantie jeunes & $\mathrm{F}$ & 22 & $\mathrm{BEP}$, début bac pro & 1 entretien & Focus group \\
\hline E34 & Léa & Destinataire Garantie jeunes & $\mathrm{F}$ & 18 & CAP & 2 entretiens & \\
\hline E35 & Sofiane & Destinataire Garantie jeunes & $\mathrm{H}$ & 22 & niveau BEPC & 2 entretiens & \\
\hline
\end{tabular}




\begin{tabular}{|c|c|c|c|c|c|c|c|}
\hline Code & Pseudo & Statut/Fonction & Sexe & Âge & Niveau d'étude & $\begin{array}{l}\text { Nombre } \\
\text { d'entretiens } \\
\text { réalisés }\end{array}$ & Autre \\
\hline E36 & Dimitri & Destinataire Garantie jeunes & $\mathrm{H}$ & NR & niveau CAP & 1 entretien & \\
\hline E37 & Julie & Destinataire Garantie jeunes & $\mathrm{F}$ & 18 & niveau CAP & 2 entretiens & \\
\hline E38 & Lara & Destinataire Garantie jeunes & $\mathrm{F}$ & 19 & niveau bac & 1 entretien & \\
\hline E39 & Jordan & Destinataire Garantie jeunes & $\mathrm{H}$ & 22 & CAP & 2 entretiens & \\
\hline E40 & Camila & Destinataire Garantie jeunes & $\mathrm{F}$ & 19 & niveau CAP & 1 entretien & Focus group \\
\hline E41 & Salamata & Destinataire Garantie jeunes & $\mathrm{F}$ & 19 & collège & 2 entretiens & \\
\hline E42 & Asta & Destinataire Garantie jeunes & $\mathrm{F}$ & 23 & BEP & 1 entretien & \\
\hline E43 & Wendy & Destinataire Garantie jeunes & $\mathrm{F}$ & 19 & sans diplôme, arrêt collège & 1 entretien & \\
\hline E44 & Abdia & Destinataire Garantie jeunes & $\mathrm{F}$ & 18 & niveau seconde & 1 entretien & \\
\hline E45 & Daouda & Destinataire Garantie jeunes & $\mathrm{H}$ & 19 & niveau $3^{\mathrm{e}}$ & 1 entretien & \\
\hline E46 & Cindy & Destinataire Garantie jeunes & $\mathrm{F}$ & 22 & niveau $3^{\mathrm{e}}$ & 2 entretiens & \\
\hline E47 & Lucas & Destinataire Garantie jeunes & $\mathrm{H}$ & 20 & niveau bac & 2 entretiens & \\
\hline E48 & Manon & Destinataire Garantie jeunes & $\mathrm{F}$ & 20 & niveau bac & 2 entretiens & Focus group \\
\hline E49 & Anthony & Destinataire Garantie jeunes & $\mathrm{H}$ & 24 & niveau $4^{\mathrm{e}}$ & 2 entretiens & \\
\hline E50 & René & Destinataire Garantie jeunes & $\mathrm{H}$ & 21 & CAP & 2 entretiens & Focus group \\
\hline E51 & Manuel & Destinataire Garantie jeunes & $\mathrm{H}$ & 22 & niveau CAP & 1 entretien & \\
\hline E52 & Nora & Destinataire Garantie jeunes & $\mathrm{F}$ & 18 & bac & 1 entretien & \\
\hline E53 & Sélim & Destinataire Garantie jeunes & $\mathrm{H}$ & 23 & CAP & 2 entretiens & \\
\hline E54 & Bintou & Destinataire Garantie jeunes & $\mathrm{F}$ & 23 & niveau BEP & 2 entretiens & \\
\hline E55 & Jacques & Destinataire Garantie jeunes & $\mathrm{H}$ & 23 & niveau $3^{\mathrm{e}}$ & 2 entretiens & \\
\hline E56 & Emilie & Destinataire Garantie jeunes & $\mathrm{F}$ & 22 & CAP & 2 entretiens & \\
\hline E57 & Ibrahim & Destinataire Garantie jeunes & $\mathrm{H}$ & 21 & niveau bac & 1 entretien & \\
\hline E58 & Asma & Destinataire Garantie jeunes & $\mathrm{F}$ & 19 & bac pro (en cours) & 1 entretien & \\
\hline E59 & Vincent & Destinataire Garantie jeunes & $\mathrm{H}$ & 23 & BEP & 2 entretiens & \\
\hline E60 & Pétronille & Destinataire Garantie jeunes & $\mathrm{F}$ & 20 & bac pro & 1 entretien & \\
\hline M1 & $* * *$ & DGEFP1 & $* * *$ & & & 1 entretien & \\
\hline M2 & $* * *$ & DGEFP2 & $* * *$ & & & 1 entretien & \\
\hline M3 & $* * *$ & DGEFP3 & $* * *$ & & & 1 entretien & \\
\hline M4 & $* * *$ & DGEFP4 & $* * *$ & & & & \\
\hline ML1 & Thérèse & $\begin{array}{l}\text { Directrice mission locale } \\
\text { violette }\end{array}$ & $\mathrm{F}$ & & & 1 entretien & \\
\hline ML2 & Cécile & $\begin{array}{l}\text { Directrice mission locale } \\
\text { verte }\end{array}$ & $\mathrm{F}$ & & & 1 entretien & \\
\hline ML3 & Claire & $\begin{array}{l}\text { Directrice-adjointe mission } \\
\text { locale verte }\end{array}$ & $\mathrm{F}$ & & & 1 entretien & \\
\hline ML4 & Françoise & $\begin{array}{l}\text { Directrice mission locale } \\
\text { orange }\end{array}$ & $\mathrm{F}$ & & & 1 entretien & \\
\hline ML5 & Adiba & $\begin{array}{l}\text { Directrice mission locale } \\
\text { jaune }\end{array}$ & $\mathrm{F}$ & & & 1 entretien & \\
\hline ML7 & Benjamin & $\begin{array}{l}\text { Conseiller mission locale } \\
\text { verte (responsable Garantie } \\
\text { jeunes) }\end{array}$ & M & & & 1 entretien & $\begin{array}{l}\text { Entretien } \\
\text { collectif et } \\
\text { observation } \\
\text { in situ }\end{array}$ \\
\hline
\end{tabular}


Une jeunesse (in)visible : les « Neets vulnérables » de la Garantie jeunes

\begin{tabular}{|c|c|c|c|c|c|}
\hline Code & Pseudo & Statut/Fonction & Sexe Âge Niveau d'étude & $\begin{array}{l}\text { Nombre } \\
\text { d'entretiens } \\
\text { réalisés }\end{array}$ & Autre \\
\hline ML8 & Camille & $\begin{array}{l}\text { Chargée de relation } \\
\text { entreprise mission locale } \\
\text { verte }\end{array}$ & $\mathrm{F}$ & 1 entretien & $\begin{array}{l}\text { Entretien } \\
\text { collectif et } \\
\text { observation } \\
\text { in situ }\end{array}$ \\
\hline ML9 & Jean & $\begin{array}{l}\text { Conseiller mission locale } \\
\text { verte }\end{array}$ & M & 1 entretien & $\begin{array}{l}\text { Entretien } \\
\text { collectif et } \\
\text { observation } \\
\text { in situ }\end{array}$ \\
\hline \multicolumn{2}{|c|}{ ML10 Kadija } & $\begin{array}{l}\text { Assistante administrative } \\
\text { mission locale verte }\end{array}$ & $\mathrm{F}$ & & $\begin{array}{l}\text { Entretien } \\
\text { collectif et } \\
\text { observation } \\
\text { in situ }\end{array}$ \\
\hline \multicolumn{2}{|c|}{ ML11 Mohand } & $\begin{array}{l}\text { Conseiller mission locale } \\
\text { violette }\end{array}$ & M & 1 entretien & \\
\hline \multicolumn{2}{|c|}{ ML12 Sofia } & $\begin{array}{l}\text { Conseillère mission locale } \\
\text { violette }\end{array}$ & $\mathrm{F}$ & 1 entretien & \\
\hline \multicolumn{2}{|c|}{ ML13 Jacques } & $\begin{array}{l}\text { Conseiller mission locale } \\
\text { orange }\end{array}$ & M & 1 entretien & $\begin{array}{l}\text { Entretien } \\
\text { collectif et } \\
\text { observation } \\
\text { in situ }\end{array}$ \\
\hline \multicolumn{2}{|c|}{ ML14 Sylvie } & $\begin{array}{l}\text { Conseillère mission locale } \\
\text { orange }\end{array}$ & $\mathrm{F}$ & 1 entretien & $\begin{array}{l}\text { Entretien } \\
\text { collectif et } \\
\text { observation } \\
\text { in situ }\end{array}$ \\
\hline
\end{tabular}


